\title{
The Twinning Rates by Zygosity in Japan, 1975-1994
}

\author{
Y. Imaizumi ${ }^{1}$, K. Nonaka ${ }^{2}$ \\ 'National Institute of Population and Social Security Research, Ministry of Health and \\ Welfare, Kasumigaseki, Chiyoda-ku, Tokyo, Japan; ${ }^{2}$ Department of Hygiene, Teikyo \\ University, School of Medicine, Kaga, Itabasiku, Tokyo, Japan
}

\begin{abstract}
The monozygotic (MZ) twinning rate in Japan had remained nearly constant from 1975 (3.74 per 1,000 births) to 1994 (4.23), whereas the dizygotic (DZ) twinning rate had remained nearly constant from 1975 (1.86) to 1986 (2.27), and had gradually increased up to 1994 (3.89). The higher DZ twinning rate since 1987 has been attributed to the higher proportion of mothers treated with ovulation-inducing hormones and partially attributed to in-vitro fertilisation in Japan. As for maternal age, $\mathrm{MZ}$ twinning rates have remained nearly constant for maternal age groups except the youngest and the oldest age groups. On the other hand, DZ twinning rates increased up to the 35-39 years of age group and decrease thereafter. In 1994, the twinning rate was higher in DZ twins than in MZ twins for maternal age groups of 30-34 years and of 35-39 years. As for geographical variations in twinning rates, DZ rates statistically significantly increased with the year during the period from 1986 to 1994 in 31 out of 47 prefectures. In 1994, twinning rates in 21 out of 47 prefectures were higher in the $\mathrm{DZ}$ than the $\mathrm{MZ}$ rate, and the $\mathrm{DZ}$ rate is equal to the $\mathrm{MZ}$ rate in two prefectures. Geographical variations in twinning rates by zygosity in 1994 drastically changed from those during the period from 19551959 and in 1974.
\end{abstract}

Key words: Twinning rates, Zygosity, Maternal age, Geographical variations

\section{INTRODUCTION}

It is well-known that the use of ovulation-inducing hormones brings a high rate of multiple births [15]. In Japan ovulation-inducing hormones have been used since 1966 [13], and since January 1975 the use of HMG (human menopausal gonadotropin) has been covered by the health insurance system. Bromocriptine has been commercially available since 1979. In a nation-wide survey in Japan, Kurachi et al. [8] examined the effect on the multiple birth rate of the ovulation-inducing hormones (combined use of HMG and 
human chorionic gonadotropin (HCG)) during the period from 1975 to 1979 . The multiple birth rate following treatment with HMG-HCG therapy was $20.5 \%$ where the rates were $13 \%$ for twins, $4.4 \%$ for triplets, $1.76 \%$ for quadruplets, $1.10 \%$ for quintuplets, and $0.22 \%$ for sixtuplets.

In Japan, the first in-vitro fertilised baby was born in 1983. A survey of in vitro fertilisation (IVF) was conducted on December 31, 1986 through questionnaires [11] and it found that among the 142 in-vitro fertilised pregnancies, $11(8 \%)$ were multiple pregnancies. According to another survey of the IVF done in 1991 [12], $290(21 \%)$ of the 1,349 in-vitro fertilised pregnancies were multiple pregnancies.

In Japan, multiple birth rates have been increasing since 1975. For example, quadruplet rates have been increasing since 1974 [3,5], triplet rates since 1975 [3], and twinning rates since 1987 [4,5]. Imaizumi [5] mentioned that the higher rate of multiple births since 1987 has been attributed to the higher proportion of mothers treated with ovulation-inducing hormones and partially attributed to in-vitro fertilisation in Japan. According to Kuroki et al. [9], the dizygotic (DZ) twinning rate in Kanagawa Prefecture was 1.6 times higher in 1991 than in 1982. They mentioned that the higher DZ rate may be attributed to the spread of treatment with ovulation-inducing hormones.

The present study focuses attention on recent trends in twinning rates and geographical variations of twinning rates by zygosity in Japan. It also deals with the effects of maternal age on zygotic twinning rates.

\section{MATERIALS AND METHODS}

Vital statistics data on twin births in Japan have been available in computer files since 1968, and in this study the computerised data between 1975 and 1994 were analysed. The vital statistics include information on parental ages, place of residence, sex of twin births according to live birth or fetal death, date of birth or fetal death, period of gestation, occupation of the head of household, and others.

Registration of births (live births and stillbirths) has been made on an individual basis, thus we have more than one record for each multiple maternity. In order to identify each pair of twins we used the information on date of birth, place of residence (codes for prefecture, health centre, and city or town within the prefecture), ages of both parents, and occupation of the head of household. There were a total of 406,350 twin births (364,554 live births and 41,796 stillbirths) during that period. First we sorted out all of the records using the above key fields, then checked adjacent records for their identity. If two adjacent records were exactly identical for the used fields, they were judged as the co-twins from a pair. With this criterion we identified 193,695 pairs $(387,390$ births, or $95.3 \%$ of the total twin births), 128 births with more than two identical records, and 18,832 unpaired births. In order to obtain more pairs allowing possible errors in registration, we repeated the above pairing procedure three times for the remaining unpaired cases. At the second cycle, we excluded the occupation of the head of household, at the third cycle we allowed for maximum 10 days' difference between birth dates of cotwins; and at the fourth cycle we allowed for one year or just 10 year difference for one of the parental ages. Finally we could identify 198,924 pairs of twins ( $97.9 \%$ of the total twin births). 


\section{RESULTS}

\section{Secular changes in zygotic twins}

Twinning rates by zygosity were estimated using Weinberg's method[13], where pairs were excluded if the sex of one or both twins was unknown.

Table 1 and Figure 1 show the secular changes in the twinning rates by zygosity during the period from 1975 to 1994 . The monozygotic (MZ) twinning rate remained nearly constant during that period, whereas the DZ twinning rate remained nearly constant from 1975 (1.86 per 1,000 births) to 1985 (2.22), and gradually increased to 3.89 in 1994 .

\section{Effect of maternal age on zygotic twinning rates}

Table 2 shows annual zygotic twinning rates by maternal age during the period from 1975 to 1994 . In all years MZ twinning rates remain nearly constant for maternal ages except the youngest and the oldest maternal age groups. On the other hand, DZ twinning rates increase up to the age group of 35-39 years and decrease thereafter in all years. Figure 2 shows zygotic twinning rates by maternal ages in the years 1975-1979, 1980-1984, 1985-1989, and 1990-1994. In the latest years, DZ rates increase rapidly with maternal age up to the age group of 30-34 years and slightly increase to the age group of 35-39 years, and decreased thereafter. The twinning rates for both zygosities are nearly the same in the age groups for 30-39 years in that period.

Figure 3 shows secular changes in DZ twinning rates by maternal age during the period from 1975 to 1994 . The DZ rate in the youngest maternal age group (<20 years) remains constant during that period except the recent two years. The rates for the age groups 30-34 years and 35-39 years are nearly constant from 1975 to 1987 (the average is 2.4 and 2.6 per 1,000 births, respectively) and rapidly increases thereafter. Similarly, the DZ rate in the age groups for 20-24 and 25-29 years remains constant up to 1986 (1.7 and 2.1 , respectively) and increases thereafter. The $D Z$ rate in the age group of 40 years and over remains constant up to 1987 and increases thereafter. Ratios of DZ rates in 1994 to the period 1975-85 are about 2 for the age groups of 30 years and over, 1.7 for the age group of 25-29 years, 1.4 for the age groups of 20-24 years and the youngest age group.

\section{Geographical variation in twinning rates}

Table 3 shows DZ twinning rates according to prefecture during the period 1986-1994. DZ rates statistically significantly increase with the year during the period from 1986 to 1994 in 31 among 47 prefectures (Table 3). The highest regression coefficient is obtained in Tochigi Prefecture, but the lowest in Hokkaido. Figure 4 shows secular changes in zygotic twinning rates in Hokkaido and Tochigi prefectures during that period. In Hokkaido, the DZ rate remains constant up to 1991 , whereas the rate increases with the year in Tochigi Prefecture. DZ twinning rates in 1993 and 1994 are higher than MZ twin- 


\section{Y. Imaizumi et al.}

ning rates in Tochigi Prefecture. The MZ twinning rate remains nearly constant for both prefectures. Secular changes of DZ twinning rates for the other prefectures range between those in Hokkaido and in Tochigi Prefecture. Among 47 prefectures, the MZ rate in Miyagi Prefecture only statistically significantly increase with the year during that period.

Figure 5 shows MZ and DZ twinning rates in each prefecture in 1994. The coefficient of variations in $\mathrm{MZ}$ twinning rates and that in $\mathrm{DZ}$ twinning rates are 0.152 and 0.218 , respectively. DZ twinning rates are especially lower in the Northeast of Japan. In 1994 , twinning rates in 21 out of 47 prefectures are higher in the DZ than the MZ rates, and the $\mathrm{DZ}$ rate is equal to the $\mathrm{MZ}$ rate in two prefectures.

Table 1 - Secular change of twin deliveries and twinning rates by zygosity, 1975-1994

\begin{tabular}{|c|c|c|c|c|c|c|}
\hline \multirow[b]{2}{*}{ Year } & \multicolumn{4}{|c|}{ Twin deliveries } & \multicolumn{2}{|c|}{ Twinning rate per 1,000 births } \\
\hline & Total* & MM & MF & $\mathrm{FF}$ & $\begin{array}{l}\text { Monozygotic } \\
\text { twins }\end{array}$ & $\begin{array}{c}\text { Dizygotic } \\
\text { twins }\end{array}$ \\
\hline 1975 & 11,805 & 4,785 & 1,860 & 4,569 & 3.74 & 1.86 \\
\hline 1976 & 11,269 & 4,562 & 1,827 & 4,338 & 3.66 & 1.89 \\
\hline 1977 & 11,477 & 4,623 & 1,866 & 4,508 & 3.93 & 2.02 \\
\hline 1978 & 11,094 & 4,541 & 1,822 & 4,224 & 3.87 & 2.03 \\
\hline 1979 & 11,004 & 4,477 & 1,846 & 4,370 & 4.06 & 2.14 \\
\hline 1980 & 10,583 & 4,319 & 1,827 & 4,152 & 4.02 & 2.21 \\
\hline 1981 & 10,426 & 4,285 & 1,768 & 4,053 & 4.08 & 2.20 \\
\hline 1982 & 10,398 & 4,224 & 1,773 & 4,092 & 4.11 & 2.23 \\
\hline 1983 & 10,299 & 4,182 & 1,751 & 4,040 & 4.09 & 2.22 \\
\hline 1984 & 10,211 & 4,097 & 1,720 & 4,080 & 4.13 & 2.20 \\
\hline 1985 & 9,806 & 3,987 & 1,667 & 3,861 & 4.12 & 2.22 \\
\hline 1986 & 9,399 & 3,855 & 1,647 & 3,565 & 3.99 & 2.27 \\
\hline 1987 & 9,318 & 3,759 & 1,613 & 3,606 & 4.08 & 2.29 \\
\hline 1988 & 9,236 & 3,692 & 1,650 & 3,594 & 4.10 & 2.40 \\
\hline 1989 & 9,074 & 3,583 & 1,684 & 3,487 & 4.14 & 2.59 \\
\hline 1990 & 8,933 & 3,506 & 1,776 & 3,307 & 3.95 & 2.78 \\
\hline 1991 & 9,142 & 3,574 & 1,902 & 3,345 & 3.94 & 2.99 \\
\hline 1992 & 9,428 & 3,708 & 2,003 & 3,403 & 4.06 & 3.18 \\
\hline 1993 & 9,644 & 3,650 & 2,158 & 3,531 & 4.07 & 3.50 \\
\hline 1994 & 10,662 & 4,047 & 2,493 & 3,867 & 4.23 & 3.89 \\
\hline
\end{tabular}

* Including unknown sexes 


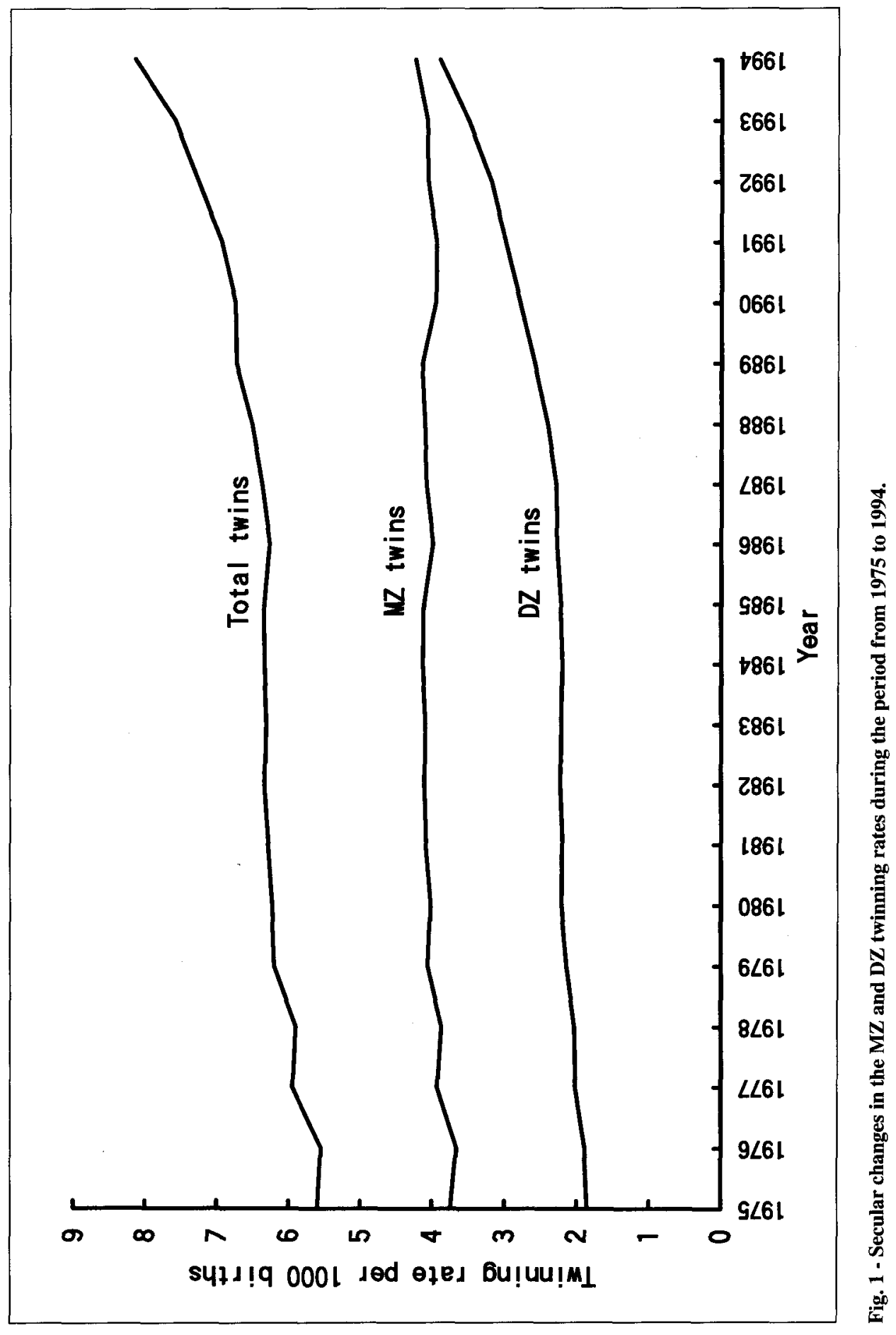




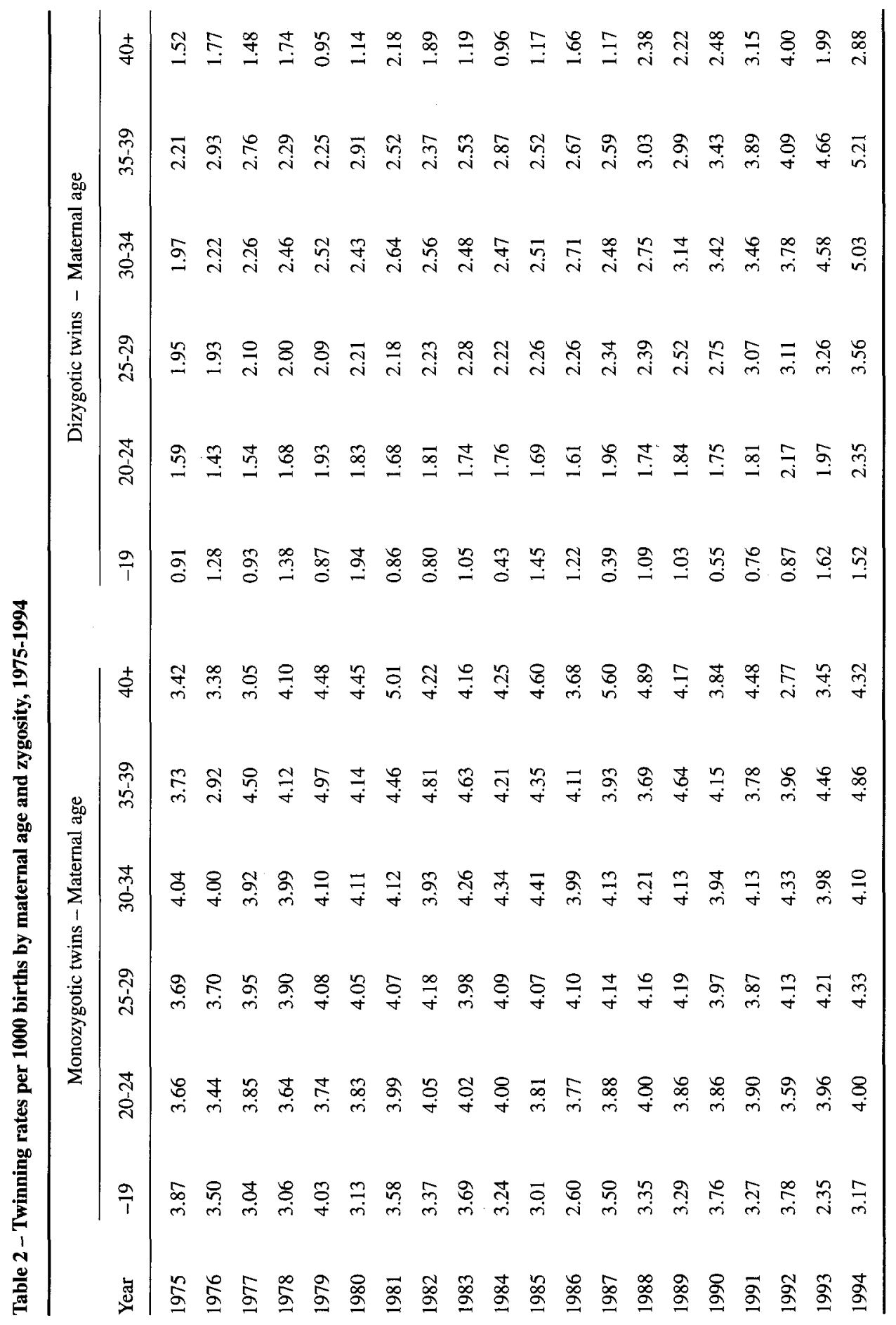




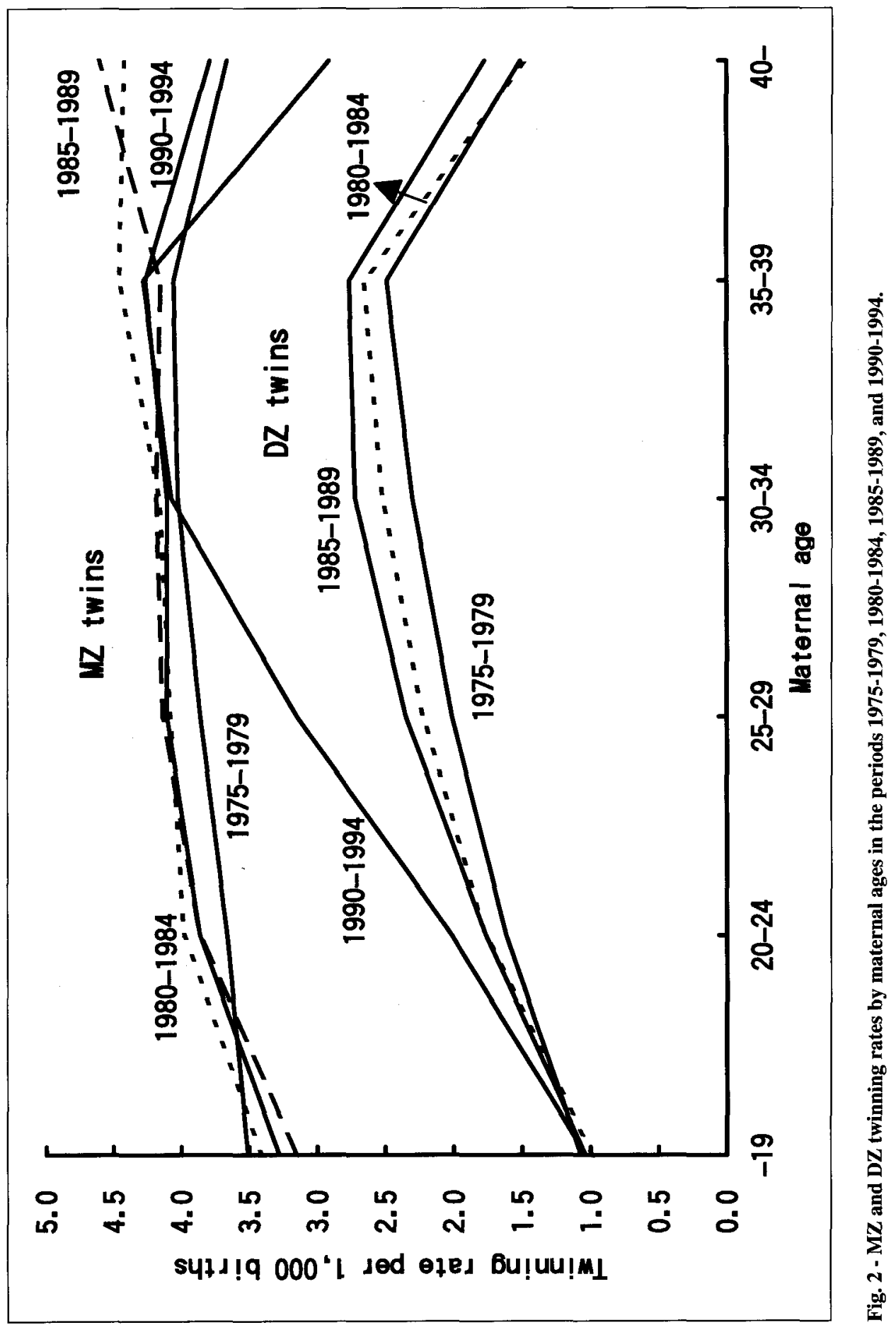




\section{Y. Imaizumi et al.}

Table 3 - Secular changes of dizygotic twinning rates in each prefecture, 1986-1994

\begin{tabular}{|c|c|c|c|c|c|c|c|c|c|c|}
\hline \multirow{2}{*}{ Prefecture } & \multicolumn{9}{|c|}{ DZ twinning rate per 1,000 births } & \multirow{2}{*}{$\begin{array}{l}\text { Regression } \\
\text { coefficient }\end{array}$} \\
\hline & 1986 & 1987 & 1988 & 1989 & 1990 & 1991 & 1992 & 1993 & 1994 & \\
\hline Hokkaido & 2.36 & 2.63 & 2.63 & 2.21 & 2.80 & 2.31 & 3.14 & 2.65 & 3.20 & 0.076 \\
\hline Aomori & 2.18 & 0.99 & 1.75 & 1.60 & 1.44 & 2.44 & 2.91 & 2.55 & 2.87 & $0.177 *$ \\
\hline Iwate & 3.33 & 2.80 & 2.10 & 3.79 & 3.88 & 4.82 & 3.02 & 3.69 & 4.07 & 0.141 \\
\hline Miyagi & 2.03 & 1.73 & 2.19 & 2.05 & 2.52 & 2.70 & 2.07 & 2.79 & 3.11 & $0.132 * *$ \\
\hline Akita & 2.16 & 2.24 & 2.37 & 2.47 & 3.30 & 3.38 & 1.97 & 4.80 & 2.51 & 0.153 \\
\hline Yamagata & 3.63 & 2.31 & 2.06 & 2.94 & 3.08 & 4.62 & 2.72 & 4.40 & 4.61 & 0.219 \\
\hline Fukushima & 2.72 & 2.49 & 2.22 & 2.96 & 3.03 & 2.27 & 3.60 & 4.07 & 4.43 & $0.228 *$ \\
\hline Ibaraki & 2.30 & 2.47 & 2.58 & 2.37 & 2.94 & 2.45 & 3.32 & 3.40 & 3.41 & $0.147 * *$ \\
\hline Tochigi & 1.77 & 2.70 & 2.84 & 3.21 & 3.26 & 4.25 & 4.05 & 5.48 & 5.86 & $0.469 * *$ \\
\hline Gunma & 2.24 & 2.95 & 3.12 & 3.44 & 3.96 & 4.19 & 3.63 & 3.73 & 5.44 & $0.281 * *$ \\
\hline Saitama & 2.36 & 2.12 & 2.69 & 3.26 & 2.56 & 2.82 & 3.14 & 3.42 & 3.45 & $0.146 * *$ \\
\hline Chiba & 2.10 & 2.11 & 2.11 & 2.67 & 2.42 & 2.42 & 3.34 & 2.61 & 3.22 & $0.137 * *$ \\
\hline Tokyo & 2.15 & 2.39 & 2.26 & 2.67 & 2.84 & 2.60 & 3.30 & 2.91 & 3.21 & $0.130 * *$ \\
\hline Kanagawa & 2.47 & 2.15 & 2.18 & 1.80 & 2.26 & 2.82 & 2.79 & 3.34 & 3.76 & $0.183 * *$ \\
\hline Niigata & 2.59 & 3.08 & 2.35 & 2.75 & 2.97 & 3.61 & 3.96 & 4.63 & 4.83 & $0.294 * *$ \\
\hline Toyama & 2.64 & 2.27 & 2.62 & 2.44 & 2.49 & 2.51 & 4.49 & 3.59 & 2.85 & 0.144 \\
\hline Ishikawa & 1.92 & 3.41 & 3.91 & 1.98 & 3.16 & 3.07 & 1.86 & 5.29 & 4.56 & 0.220 \\
\hline Fukui & 2.18 & 2.77 & 2.71 & 3.20 & 2.23 & 2.94 & 2.09 & 3.51 & 4.03 & 0.135 \\
\hline Yamanashi & 2.35 & 2.58 & 2.95 & 1.96 & 2.24 & 2.15 & 1.52 & 3.73 & 3.98 & 0.122 \\
\hline Nagano & 3.13 & 2.95 & 1.96 & 2.56 & 3.34 & 3.37 & 3.33 & 3.58 & 4.69 & $0.195 *$ \\
\hline Gifu & 2.07 & 2.24 & 2.29 & 2.34 & 2.66 & 2.31 & 3.33 & 3.87 & 3.01 & $0.178 * *$ \\
\hline Shizuoka & 2.21 & 2.56 & 2.08 & 2.32 & 3.53 & 2.84 & 3.97 & 3.27 & 4.71 & $0.274 * *$ \\
\hline Aichi & 2.43 & 2.05 & 2.40 & 3.01 & 2.95 & 2.91 & 3.23 & 3.63 & 4.01 & $0.210 * *$ \\
\hline Mie & 2.06 & 2.40 & 2.75 & 2.33 & 3.01 & 2.42 & 2.73 & 2.01 & 4.17 & 0.121 \\
\hline Shiga & 1.72 & 2.82 & 2.58 & 2.15 & 2.13 & 2.63 & 3.64 & 4.63 & 3.77 & $0.270 *$ \\
\hline Kyoto & 1.48 & 1.87 & 2.93 & 3.48 & 2.93 & 3.19 & 3.39 & 3.68 & 4.15 & $0.279 * *$ \\
\hline Osaka & 2.14 & 1.86 & 2.14 & 2.26 & 2.50 & 2.96 & 2.97 & 3.45 & 3.43 & $0.204 * *$ \\
\hline Hyogo & 2.66 & 2.76 & 2.41 & 2.83 & 2.47 & 3.00 & 2.95 & 3.43 & 3.46 & $0.107 *$ \\
\hline Nara & 1.71 & 2.50 & 1.78 & 1.15 & 2.46 & 1.77 & 2.03 & 3.73 & 2.73 & 0.148 \\
\hline Wakayama & 1.29 & 2.04 & 4.23 & 2.22 & 2.84 & 2.27 & 2.33 & 1.99 & 4.96 & 0.179 \\
\hline Tottori & 2.59 & 1.91 & 2.23 & 2.99 & 3.28 & 3.42 & 3.21 & 2.65 & 6.08 & $0.309 *$ \\
\hline Shimane & 2.60 & 2.71 & 3.77 & 2.48 & 4.10 & 3.13 & 4.61 & 4.71 & 3.84 & $0.222 *$ \\
\hline Okayama & 2.27 & 1.89 & 2.81 & 3.16 & 2.71 & 3.67 & 3.27 & 4.82 & 3.74 & $0.268 * *$ \\
\hline
\end{tabular}


Table 3

\begin{tabular}{lccccccccccc}
\hline & \multicolumn{8}{c}{ DZ twinning rate per 1,000 births } & Regression \\
\cline { 2 - 8 } & 1986 & 1987 & 1988 & 1989 & 1990 & 1991 & 1992 & 1993 & 1994 & coefficient \\
\hline Hiroshima & 1.64 & 2.44 & 2.59 & 2.18 & 3.06 & 2.84 & 2.37 & 3.52 & 4.35 & $0.238^{* *}$ \\
Yamaguchi & 3.28 & 1.91 & 3.26 & 2.90 & 3.06 & 3.84 & 4.39 & 5.88 & 4.72 & $0.347 * *$ \\
Tokushima & 2.48 & 2.62 & 1.75 & 2.14 & 2.18 & 4.98 & 2.07 & 4.45 & 3.14 & 0.193 \\
Kagawa & 1.88 & 2.32 & 3.12 & 2.35 & 4.04 & 3.07 & 3.26 & 4.26 & 5.69 & $0.368 * *$ \\
Ehime & 2.31 & 2.68 & 2.51 & 3.40 & 2.74 & 3.05 & 3.86 & 2.20 & 4.90 & 0.187 \\
Kochi & 2.54 & 2.48 & 1.63 & 2.25 & 3.45 & 2.84 & 3.73 & 2.86 & 3.91 & $0.190 *$ \\
Fukuoka & 1.99 & 1.90 & 2.82 & 3.09 & 2.95 & 4.44 & 4.24 & 4.00 & 4.97 & $0.373 * *$ \\
Saga & 1.52 & 1.08 & 1.86 & 2.43 & 2.39 & 3.87 & 4.38 & 3.62 & 4.34 & $0.423 * *$ \\
Nagasaki & 2.16 & 1.38 & 2.06 & 2.19 & 3.33 & 2.74 & 3.20 & 4.23 & 5.51 & $0.413 * *$ \\
Kumamoto & 1.85 & 2.18 & 2.19 & 2.31 & 2.09 & 2.40 & 2.86 & 2.91 & 3.80 & $0.191 * *$ \\
Oita & 2.55 & 2.69 & 1.89 & 1.24 & 1.30 & 3.04 & 3.13 & 3.04 & 5.37 & 0.277 \\
Miyazaki & 1.77 & 1.21 & 1.80 & 2.77 & 2.49 & 2.33 & 3.60 & 3.07 & 4.35 & $0.317 * *$ \\
Kagoshima & 1.80 & 2.44 & 1.54 & 2.31 & 2.70 & 2.79 & 1.37 & 3.03 & 3.94 & 0.175 \\
Okinawa & 3.23 & 2.98 & 2.05 & 3.09 & 2.60 & 4.27 & 3.44 & 4.50 & 2.78 & 0.112 \\
\hline
\end{tabular}

* Significant at the $5 \%$ level; $\quad * *$ Significant at the $1 \%$ level.

\section{DISCUSSION}

Imaizumi and Inouye [6] reported the secular trends of the twinning rate according to zygosity during the period from 1955 to 1967 and in 1974 in Japan. The rates of MZ and DZ twin births slightly decreased during that period. In the present study, the MZ rate remained nearly constant during the period from 1975 to 1994 , whereas the DZ rate remained nearly constant up to 1986, and gradually increased up to 1994. Similarly, using vital statistics data on twin births during the period from 1988 to 1991 in Japan, Asaka et al. [1] mentioned that the proportion of dizygotic twins among total twin pairs increased during that period.

From the Survey on Socio-Economic Aspects of Vital Events-Plural Births in 1975 [7], the proportion of mothers treated with ovulation-inducing hormone was higher in mothers of unlike-sexed twins $(9.2 \%, 72 / 786)$ than in those of like-sexed twins $(4.5 \%, 158 / 3485)(1979)$. As mentioned previously, the proportion of twins following treatment with HMG-HCG therapy was 13\%[8]. Recently, HMG (or hMG) has been used for the IVF [10]. The number of multiple births by IVF increased yearly [5]. Therefore, the higher DZ twinning rate since 1987 has been attributed to the higher proportion of mothers treated with ovulation-inducing hormones and partially attributed to in-vitro fertilisation in Japan.

For the period from 1990 to $1994, \mathrm{MZ}$ and DZ rates took similar values for the 
Y. Imaizumi et al.

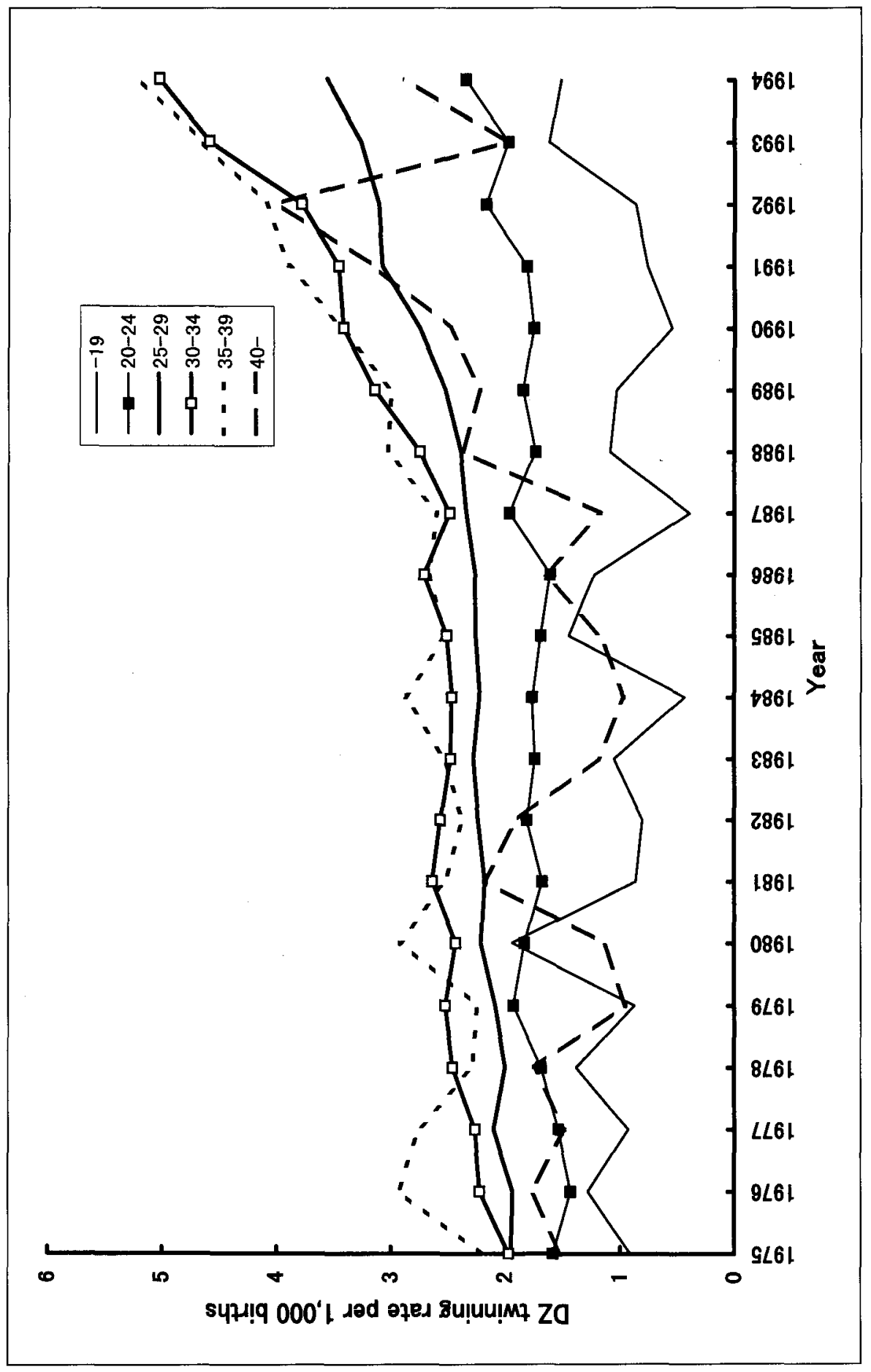

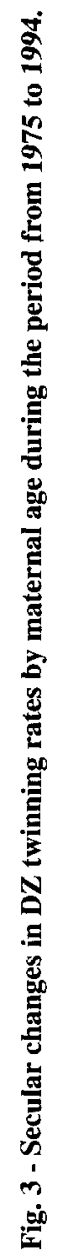


maternal age groups 30-34 years and 35-39 years. It seems that the proportion of mothers treated with ovulation-inducing hormones or in-vitro fertilization was the highest for the maternal age groups 30-34 years and followed by the maternal age groups 35-39 years. For the recent years, mothers of age groups 25-29 years and over the age of 40 years are treated with ovulation-inducing hormones or in-vitro fertilisation.

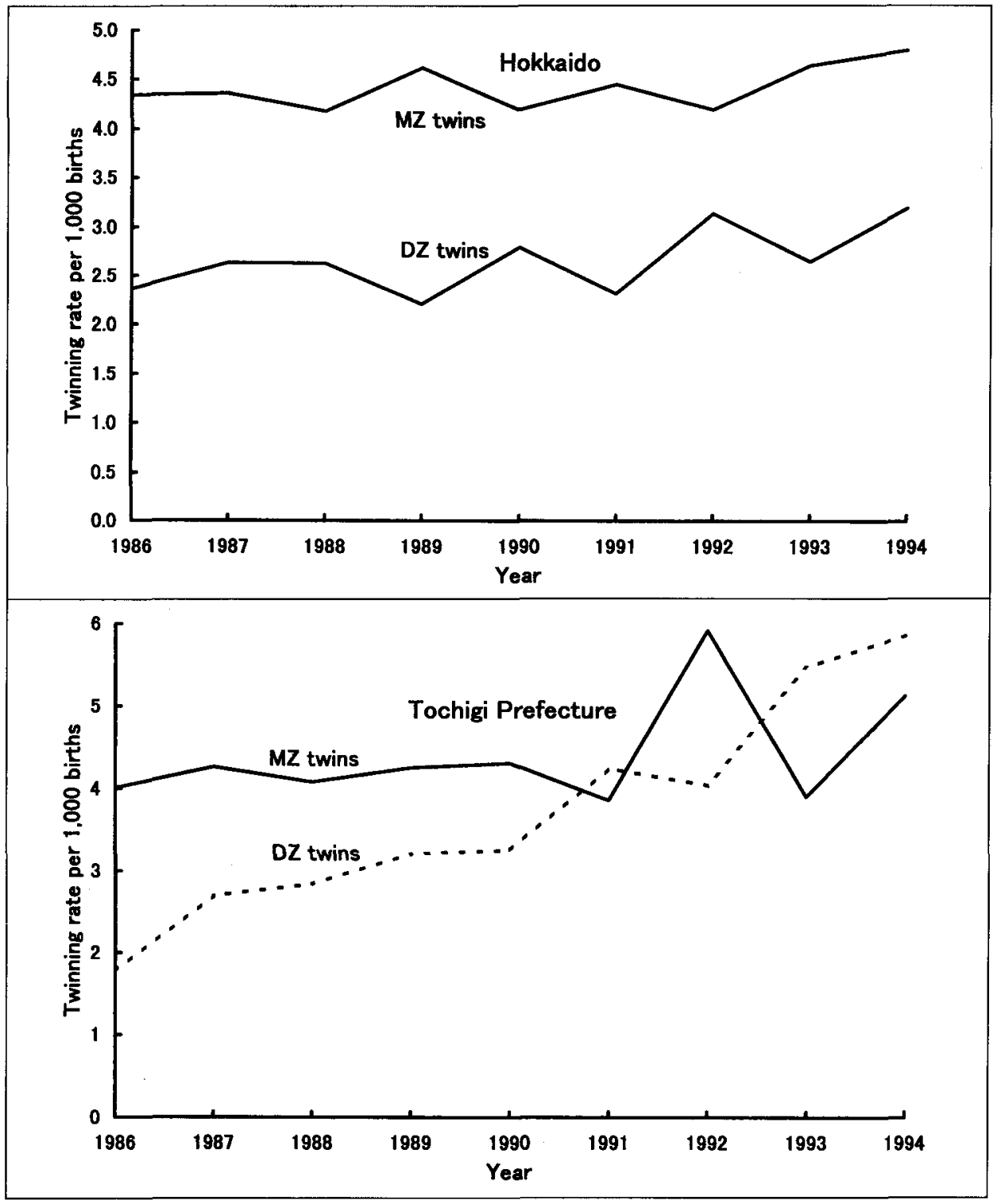

Fig. 4 - Secular changes in MZ and DZ twinning rates in Hokkaido and Tochigi prefectures during the period 1986-1994. 


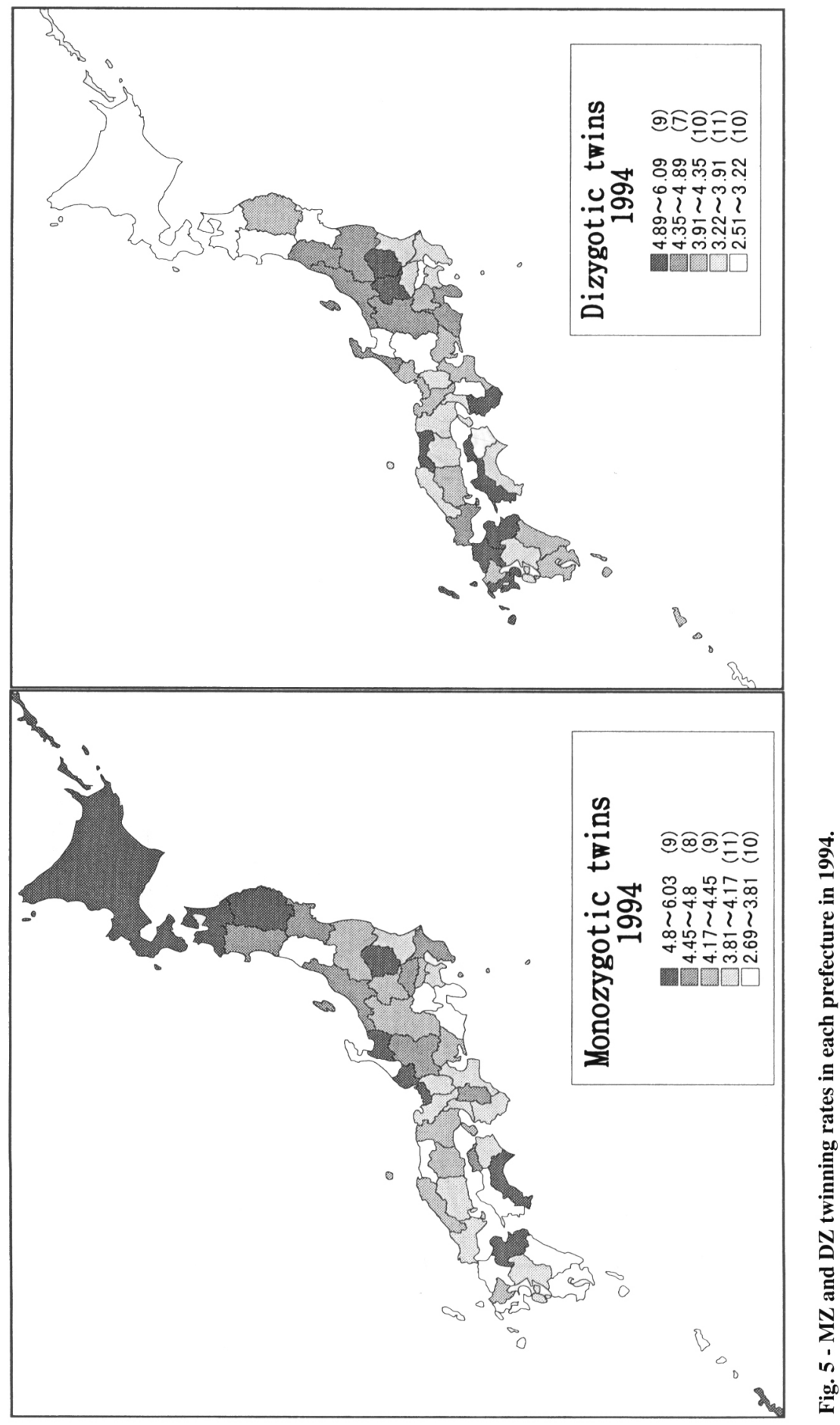


As for geographical variations in the DZ twinning rate, Imaizumi and Inouye [6] mentioned that the MZ rate was rather constant throughout Japan in 1974, whereas the $\mathrm{DZ}$ rate increased from a low level in the Southwest of Japan to a high level in the Northeast, except for the Okinawa Prefecture located on the Southwest tip of Japan. Similar results were also obtained during the period from 1955 to 1959 in Japan [4]. On the other hand, in the present study, the MZ rate was not constant throughout Japan in 1994 (Fig. 4). The DZ rate was low in the Northeast than in the Southwest of Japan, except for the Okinawa Prefecture in 1994. Geographical variations in twinning rates by zygosity in 1994 drastically changed from those in 1974. The Japanese Working Group for Registrations on Assisted Reproduction asked 354 maternity clinical institutions whether to register or not in 1995 [2]. Among which 103 institutions accepted the request. These addresses were obtained from the report [2], but these are only under one third of the 364 institutions in Japan. Therefore, a relationship between the DZ twinning rate and the proportion of the number of such maternity clinical institutions per population in each prefecture was not obtained. However, it seems that there is a relationship between a higher $\mathrm{DZ}$ twinning rate and the number of popular clinical institutions in a few prefectures including the neighbouring ones (Tochigi, Fukuoka Prefectures etc.).

Acknowledgement: We are grateful to the staff, Health and Welfare Statistics and Information Department, Ministry of Health and Welfare. This study was supported in part by the Grant Aided by the Ministry of Health and Welfare of Japan for the Handicapped Children, 1994-1997.

\section{REFERENCES}

1. Asaka A, Kato N, Ooki S, Amou Y (1996): Increasing proportion of dizygotic twins among total twin births. In Terao T, ed. Study on management and care for multiple pregnancies, the Ministry of Health and Welfare of Japan, Tokyo, 1995: 207-210 (in Japanese).

2. Hiroi M, Ibuki R, Noda Y, et al. (1996): The Committee Report on the Registration for Reproductive Medicine in 1994 (the 7th report). Acta Obst Gynaec Jap, 48: 1182-1196.

3. Imaizumi Y (1987): The recent trends in multiple births and stillbirth rates in Japan. Acta Genet Med Gemellol 36: 325-334.

4. Imaizumi Y (1992): Twinning rates in Japan, 1951-1990. Acta Genet Med Gemellol 41: 165- 175.

5. Imaizumi Y (1994): Recent and long term trends of multiple birth rates and influencing factors in Japan. J of Epidemiology 4: 103-109 (1994).

6. Imaizumi Y, Inouye E (1979): Analysis of multiple birth rates in Japan. I. Secular trend, maternal age effect, and geographical variation in twinning rates. Acta Genet Med Gemellol 28: 107124.

7. Japan, Ministry of Health and Welfare: Survey on Socio-Economic Aspects of Vital EventsPlural Births. 1975. Health and Welfare Statistics and Information Department, Ministry of Health and Welfare, Tokyo, 1977 (in Japanese).

8. Kurachi, $\mathrm{K}$ et al (1983): The outcome of pregnancy following medical treatment to the HMG (Humegon)-HCG. Sanka to Fujinka 50: 274-281.

9. Kuroki Y, Imaizumi K, Tsunoda A, Fujii A, Konishi H (1992): Survey on the monitoring of congenital anomalies in Kanagawa Prefecture. In Takano A, ed. Study on effects of community and family environment on children, the Ministry of Health and Welfare of Japan, Tokyo, 1992: 65-68 (in Japanese). 


\section{Y. Imaizumi et al.}

10. Mizunuma H, Igarashi M (1991): Ovulation-inducing hormones (HMG): Its theory and practice. Obsterical and Gynecological Practice 40: 321-327 (in Japanese)

11. Mori T (1987): Progress in reproductive and medical technology. Sanfujinka no Sekai 39: 963966 (in Japanese).

12. Mori T, Aono T, Shimizu T, et al. (1993): The committee Report on the Registration for Reproductive Medicine in 1992 (the 4th report). Acta Obst Gynaec Jap, 45: 397-410 (in Japanese).

13. Sawazaki C, Tsubata H (1976): Statistics of multiple births in Japan. Sanka to Fujinka 43: 863869 (in Japanese).

14. Weinberg W (1901): Beitrage zur Physiologie und Pathologie der Mehrlingsgeburten beim Menschen. Arch Ges Physiol 88: 346-430.

15. Wyshak G (1978): Statistical findings on the effects of fertility drugs on plural births. In Nance WE, Allen G, Parisi P (eds): Twin Research: Part B, Biology and Epidemiology. New York: Alan R. Liss, p 17-33.

Correspondence: Dr. Ioko Imaizumi, National Institute of Population and Social Security Research, 1-2-

3, Kasumigaseki, Chiyoda-ku, Tokyo 100, Japan 